\title{
INVAGINACION COLO-COLICA: PRESENTACIÓN DE DOS CASOS
}

\author{
Interno Jorge Llanos $C^{(1)}$, Drs. Claudia Ortega $M^{(2)}$, Raúl Rojas $C^{(2)}$, Andrés O'Brien $\mathbf{S}^{(2)}$.
}

1. Interno. Facultad de Medicina. Universidad Católica de Chile

2. Servicio de Radiología, Hospital Clínico Pontificia Universidad Católica de Chile.

Abstract: Intussusception is an uncommon event in adults, with different clinical presentations, which range from bowel obstruction symptoms to nonspecific abdominal pain. Can occur in the small or large bowel. In adults there is usually an underlying cause, usually benign or malignant neoplasms, being the latter the main cause of this disease in the colon. The diagnosis with CT is usually straightforward, with a very typical appearance. We report two cases of patients with bowel obstruction and diagnosis of intussusception caused by a lipoma and colon cancer respectively.

Keys word: Colic lipoma, Colon cancer, Intussusception.

Resumen: El fenómeno de invaginación en el adulto es un evento poco frecuente, con una amplia variedad de presentación, que va desde síntomas agudos de obstrucción intestinal, hasta síntomas abdominales crónicos inespecíficos. Puede ocurrir tanto en intestino delgado como colon, siendo frecuente encontrar lesiones orgánicas subyacentes, en su mayoría procesos neoplásicos, tanto benignos como malignos Estos últimos son la etiología principal de invaginaciones en intestino grueso. El diagnóstico se puede realizar de forma confiable con el uso de tomografía computada al identificarse características imagenológicas particulares a esta patología. Reportamos dos casos en pacientes que se presentan con síntomas de obstrucción intestinal, con lesiones subyacentes en ambos casos, un lipoma y una neoplasia maligna.

Palabras claves: Cáncer de colon, Intususcepción, Lipoma cólico.

Llanos J, y cols. Invaginación colo-colica: Presentación de dos casos. Rev Chil Radiol 2005; 11: 9-12. Correspondencia:rfrojas@puc.cl·cbortega@puc.cl

\section{Introducción}

El fenómeno de invaginación se produce cuando un segmento, generalmente proximal, del intestino llamado intususceptum se desplaza al lumen de un segmento intestinal adyacente distal llamado intususcipiens. Es una patología rara en adultos y las colo-cólicas comprenden el $5 \%$ del total de invaginaciones, causando el $1 \%$ de todas las obstrucciones intestinales y $0.003-0.02 \%$ de los ingresos hospitalarios ${ }^{(1)}$. Se describe que el $80-90 \%$ del total de las invaginaciones en adultos tienen una causa subyacente, en su mayoría procesos benignos.

\section{Caso clínico 1}

Paciente de 46 años, del sexo femenino, previamente sana, quien consulta en el Servicio de Urgencia por un cuadro de dolor abdominal de dos semanas de evolución localizado en fosa iliaca izquierda, sin presentar fiebre en su evolución. Al examen físico presenta sensibilidad en el hemiabdomen izquierdo, sin signos de irritación peritoneal, no detectándose una masa palpable.

Se solicita TC de abdomen y pelvis en la cual se demostró una invaginación colo-cólica cuyo extremo se identifica a nivel del ángulo esplénico, observándose en este una masa con densidad grasa, de aproximadamente $10 \mathrm{cms}$. de longitud, compatible con un lipoma intraluminal (Figura 1).

Se efectuó colonoscopía (Figura 2) y posterior a ella colectomía parcial, el estudio anatomopatológico, confirmó el diagnostico de lipoma.

\section{Caso clínico 2}

Paciente de 65 años, del sexo masculino, con historia de dolor abdominal epigástrico y en hipocondrio derecho, de varias semanas de evolución, sin baja de peso ni otros signos de alerta.

Se realiza ecotomografía abdominal y TC de abdomen (Figura 3 ) que demostraron una invaginación con una masa en su extremo ubicada a 

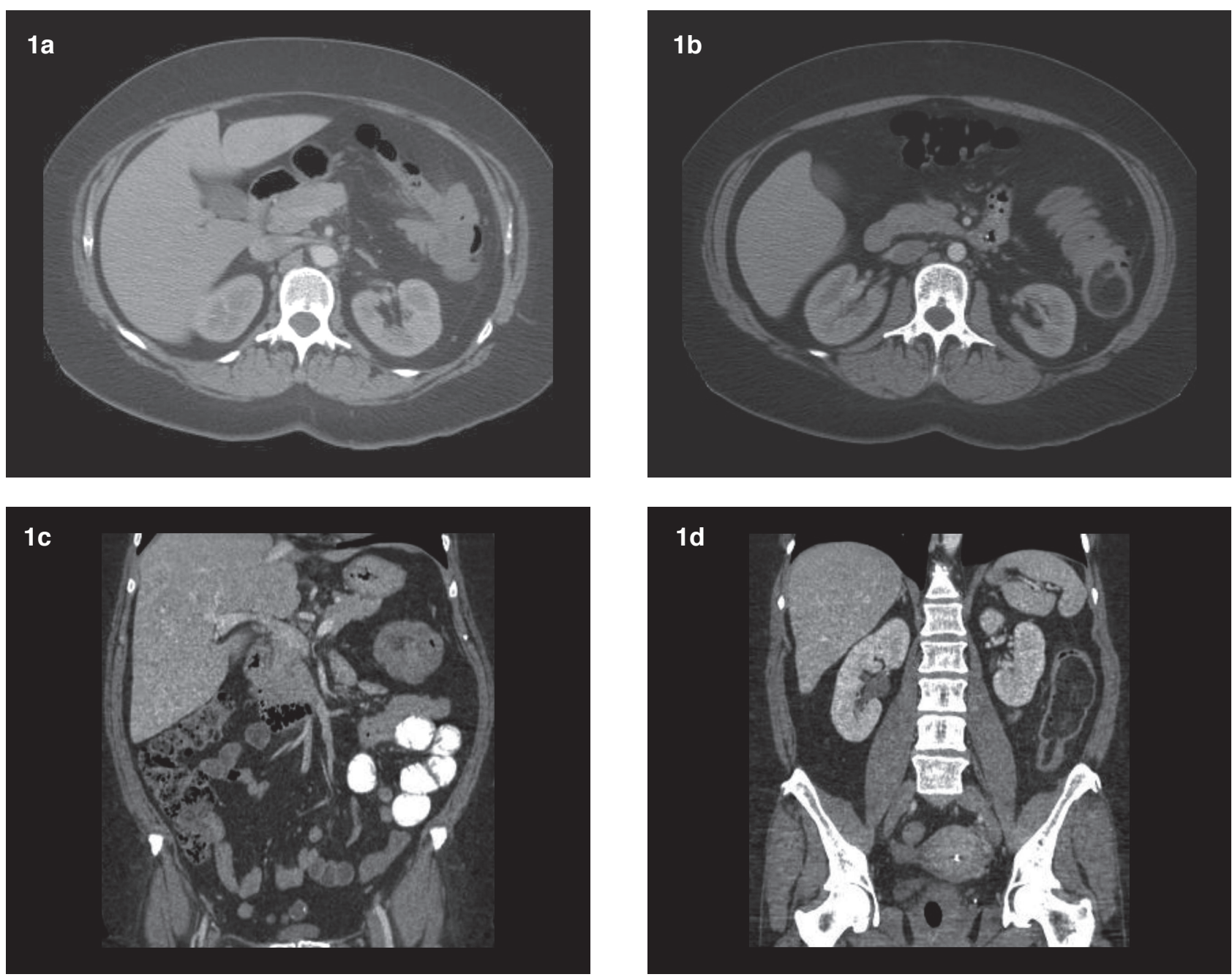

Figura 1 a-d. TC de abdomen y pelvis cortes axiales (a,b) y reconstrucciones coronales (c,d). Se observa la invaginación a nivel del ángulo esplénico del colon ( $a, c)$, en el extremo de la cual se identifica una masa con densidad grasa (b,d) con los caracteres de un lipoma intraluminal.

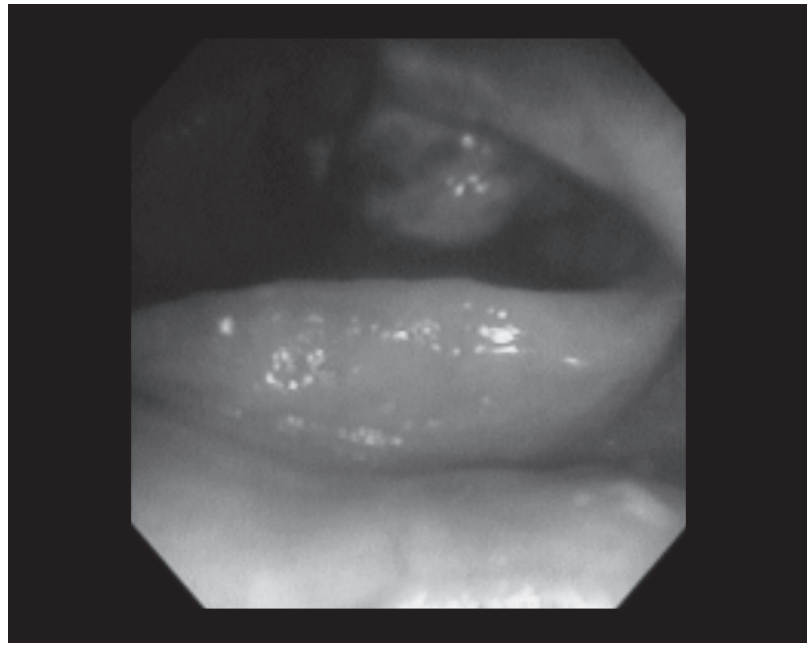

Figura 2. Colonoscopía del caso 1 en la cual se observa en la zona próximal del colon descendente una formación polipoídea de $10 \mathrm{cms}$ de longitud por 3-4 cms de ancho, compatible con lipoma. nivel del colon transverso. Se practica luego una colonoscopía que confirmó la presencia de la masa (Figura 4) y posteriormente se efectuó hemicolectomía derecha. El estudio anatomo-patológico de la pieza confirmó el diagnóstico de un adenoma velloso del intestino grueso con displasia epitelial intensa, y focos microscópicos de adenocarcinoma tubular bien diferenciado.

\section{Discusión}

La invaginación colo-cólica es una patología infrecuente en adultos y cuando se presenta debe hacer sospechar una causa subyacente, que en este tipo de invaginación corresponde en la mayoría de los casos un proceso maligno.

El mecanismo fisiopatológico de la invaginación se produce por el impulso de una masa situada en el lumen intestinal y es activada por los movimientos peristálticos normales. En la ausencia de masas se produciría por alteraciones funcionales causadas por 

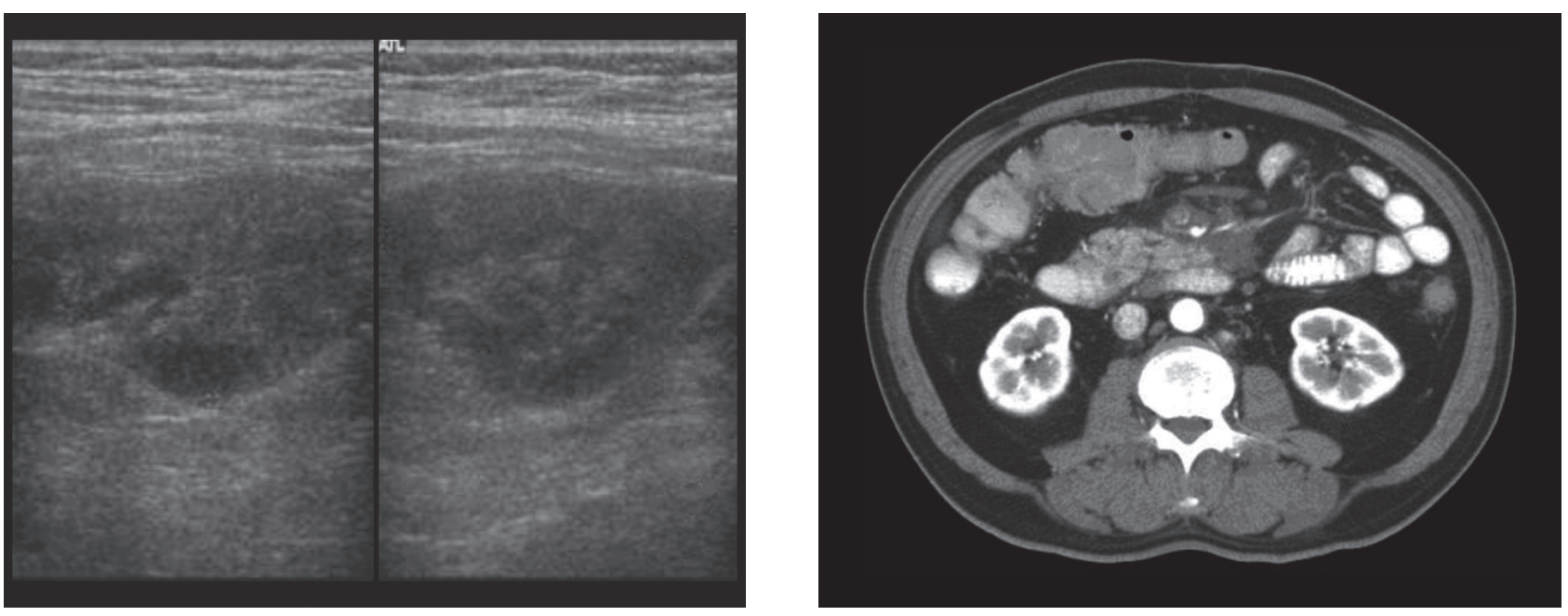

Figura 3 a,b. a: Ultrasonografía, en imagen dual, demostrando una masa hipoecogénica en flanco derecho a nivel del colon transverso con un área central de mayor ecogenicidad. b: TC demostrando que la masa corresponde a un tumor de colon transverso que ha actuado como punto guía de una invaginación.

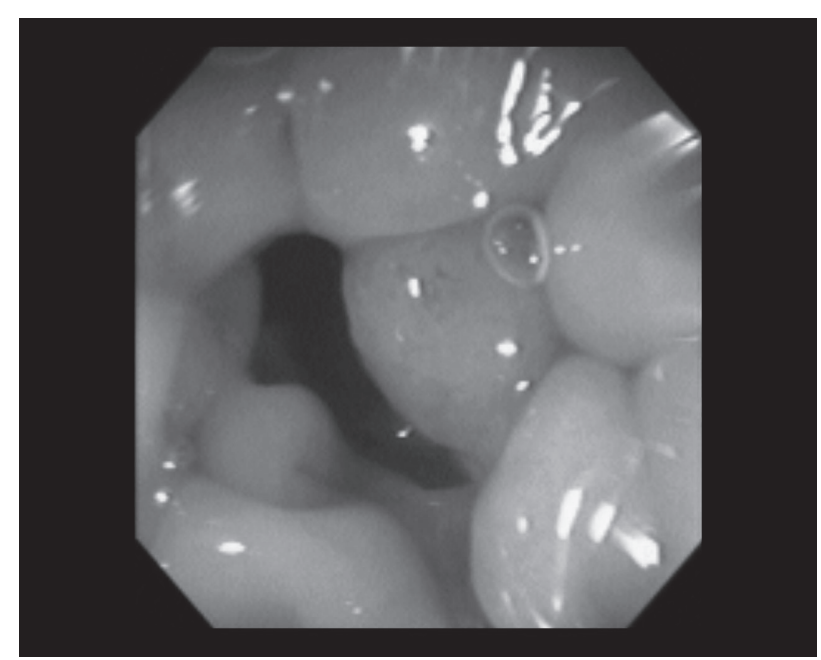

Figura 4. Colonoscopía del caso 2 que demuestra la presencia de una gran masa en el colon transverso, que ocupa el $75 \%$ del perímetro, de aspecto polipoídeo, de aproximadamente $5 \mathrm{~cm}$ de diámetro. Las muestras para estudio histopatológico confirmaron un adenoma velloso.

una enfermedad subyacente que logre alterar la pared intestinal, como ocurre en la enfermedad celiaca.

Las invaginaciones se pueden clasificar de acuerdo a su localización en entéricas, que comprometen yeyuno e íleon, y cólicas, que incluyen las íleo-cólicas y colo-cólicas. En una serie de 58 pacientes del Hospital General de Massachussets un $76 \%$ de los casos correspondieron al tipo entéricas, y un $24 \%$ a cólicas $^{(1)}$. Otra serie publicada posteriormente que incluye 33 pacientes demostró un $88 \%$ de entéricas y $12 \%$ de invaginaciones en colon ${ }^{(2)}$.

Del total de invaginaciones se identifica una lesión orgánica en un porcentaje que varia entre un 48 a un $93 \%$ de los casos, en diferentes publicaciones ${ }^{(1-3)}$.
Algunas series de casos, en donde el diagnostico se efectuó en forma quirúrgica, refieren que las causas neoplásicas producen la mayoría de las de invaginaciones, reportándose una incidencia de hasta un $66 \%$. Esta situación; sin embargo varía al usar la TC como herramienta de diagnóstico, en donde se identifican aproximadamente sólo un $1 / 3$ de lesiones neoplásicas, probablemente debido al mayor número de diagnóstico de invaginaciones transitorias, que no requieren tratamiento quirúrgico ${ }^{(1,5)}$.

El intestino delgado es el sitio donde se produce la mayoría de las invaginaciones alcanzando aproximadamente $3 / 4$ de los $\operatorname{casos}^{(1)}$, siendo la causa principal neoplasias benignas tales como lipoma, leiomioma, hemangioma, neurofibroma. También son causa de ellas, adherencias post-operatorias, enfermedad celiaca, hiperplasia linfoide y adenitis, trauma, púrpura de Schöenlein-Henoch, enfermedad celiaca, entre otras. Lesiones malignas se encuentran entre un $15-48 \%$ según sea la serie revisada ${ }^{(1,4)}$ siendo en este caso, las metástasis la causa mas frecuente.

En el colon las invaginaciones son menos frecuentes que en intestino delgado, pero al contrario de estas últimas, la causa principal en su mayoría son lesiones neoplásicas malignas constituyendo su incidencia entre un 47 a 100\% dependiendo de la serie ${ }^{(1,2,4,6)}$. Generalmente son lesiones primarias de colon, tales como el adenocarcinoma y linfoma. Causas benignas de invaginaciones en colon son algunas tumores tales como lipomas, leiomiomas y pólipos adenomatosos.

Los síntomas clínicos pueden aparecer en forma aguda o crónica, sin embargo en la mayoría de los casos son de larga duración. Los más frecuentes 
son aquellos que hacen referencia a obstrucción intestinal, tales como nauseas, vómitos, dolor abdominal y están presentes en un $78 \%$ de los pacientes. Síntomas como pérdida de peso, diarrea o masa palpable se encuentran sólo en $10 \%$ de los casos. El diagnóstico clínico de invaginación se hace en aproximadamente entre el $30-50 \%$ de los $\operatorname{casos}^{(1-6)}$.

La TC permite un diagnostico en forma confiable de las invaginaciones y se han descrito tres tipos de presentación que reflejan la duración y severidad del proceso. En general el aspecto es de una masa de tejido blando, compuesto del intususcipiens externo y del intususceptum central. Frecuentemente se observa un área excéntrica de densidad de grasa dentro de la masa, que corresponde a la grasa mesentérica invaginada, pudiendo verse los vasos mesentéricos dentro de esta. La forma más temprana de presentación es lo que se describe como «target»o «diana», observado en cortes transversales y axiales. Una segunda etapa posible de identificar es una masa con «forma de salchicha» (sausage shaped), y se ve en cortes coronales y sagitales, la cual representa la pared intestinal y grasa mesentérica. La etapa más tardía se aprecia como una masa reniforme dada por edema, engrosamiento mural y compromiso vascular. Otro hallazgo posible de identificar es un aro de contraste oral rodeando al intususceptum y representa la envoltura de las paredes intestinales opuestas ${ }^{(4,10)}$. Si bien la apariencia de la invaginación es característica en el TC, su etiología no se puede establecer con certeza a excepción del lipoma, debido a la presencia en general de hemorragia o tejido edematoso(4).

Se han determinado ciertos parámetros en TC que podrían permitir distinguir invaginaciones autolimitadas de aquellas que requerirán tratamiento quirúrgico. El principal signo considerado es la longitud de la invaginación considerándose que cuando es menor a $3.5 \mathrm{cms}$, correspondería a la forma autolimitada; la mayor validez de este signo es aplicable a invaginaciones de intestino delgado(5).

El hallazgo de un lipoma en colon como causa de invaginación es infrecuente y hasta el año 2002, se habían reportado en la literatura 32 casos secundarias a esta causa. Lipomas en colon son una patología rara en la población general, ocurren en solo el 0,2\% en grandes series de autopsias, siendo hasta un 2,6\% de neoplasias benignas gastrointestinales. En una serie de 14 invaginaciones cólicas 3 fueron causadas por lipomas. La mayoría de ellos son de localización submucosa, principalmente ubicados en el ciego y dan síntomas cuando alcanzan tamaños superiores a 2 cms., incluyendo diarrea, constipación, sangramiento e invaginación ${ }^{(1,9)}$. El lipoma se identifica en TC como una masa de densidad grasa, la cual puede verse alterada por distintos grados de infarto o necrosis, que no contiene vasos sanguíneos, imagen que se observa dentro del lumen del intususcipiente ${ }^{(4,8,9)}$.

El tratamiento óptimo para las invaginaciones en adultos no esta del todo establecido, aunque todos los autores concuerdan que la laparotomía es mandatoria por la alta probabilidad de encontrar una lesión subyacente ${ }^{(6)}$.

\section{Bibliografía}

1. Azar T, Berger DL. Adult Intussusception. Ann Surg 1997; 226: 134-138.

2. Warshauer DM, Lee JK. Adult intussusception detected at CT or MR imaging: Clinical-imaging correlation. Radiology 1999; 212: 853-860.

3. Omori $\mathrm{H}$, Asahi $\mathrm{H}$, Inoue $\mathrm{Y}$ et al. Intussusception in adults: A 21-year experience in the universityaffiliated emergency center and indication for nonoperative reduction. Dig Surg. 2003; 20: 433-9.

4. Gayer G, Zissin R, Apter S et al. Adult intussusceptiona CT diagnosis. Brit J Radiol 2002; 75: 185-190.

5. Lvoff N, Breiman RS, Coakley FV et al. Distinguishing features of self-limiting adult small-bowel intussusception identified at CT. Radiology 2003; 227: 68-72.

6. Takeuchi K, Tsuzuki Y, Ando T et al. The diagnosis and treatment of adult intussusception. J Clin Gastroenterol 2003; 36: 18-21.

7. Fujimoto T, Fukuda T, Uetani M et al. Unenhanced CT findings of vascular compromise in association with intussusceptions in adults. AJR Am J Roentgenol 2001; 176: 1167-1171.

8. Ross GJ, Amilineni V. Case 26: Jejunojejunal intussusception secondary to a lipoma. Radiology 2000; 216: 727-730.

9. Rogers SO Jr, Lee MC, Ashley SW. Giant colonic lipoma as lead point for intermittent colo-colonic intussusception. Surgery 2002; 131: 687-8.

10. Byrne AT, Geoghegan T, Govender P et al. The imaging of intussusception. Clin Radiol 2005; 60: 39-46. 